\title{
Circadian profiles in young people during the early stages of affective disorder
}

\author{
SL Naismith ${ }^{1}$, DF Hermens ${ }^{1}$, TKC Ip ${ }^{1}$, S Bolitho ${ }^{1}$, E Scott ${ }^{1}$, NL Rogers $^{2,3}$ and IB Hickie ${ }^{1}$
}

Although disturbances of the circadian system are strongly linked to affective disorders, no known studies have examined melatonin profiles in young people in early stages of illness. In this study, 44 patients with an affective disorder underwent clinical and neuropsychological assessments. They were then rated by a psychiatrist according to a clinical staging model and were categorized as having an 'attenuated syndrome' or an 'established disorder'. During the evening, salivary melatonin was sampled under dim light conditions over an 8-h interval and for each patient, the time of melatonin onset, total area under the curve and phase angle (difference between time of melatonin onset and time of habitual sleep onset) were computed. Results showed that there was no difference in the timing of melatonin onset across illness stages. However, area under the curve analyses showed that those patients with 'established disorders' had markedly reduced levels of melatonin secretion, and shorter phase angles, relative to those with 'attenuated syndromes'. These lower levels, in turn, were related to lower subjective sleepiness, and poorer performance on neuropsychological tests of verbal memory. Overall, these results suggest that for patients with established illness, dysfunction of the circadian system relates clearly to functional features and markers of underlying neurobiological change. Although the interpretation of these results would be greatly enhanced by control data, this work has important implications for the early delivery of chronobiological interventions in young people with affective disorders.

Translational Psychiatry (2012) 2, e123; doi:10.1038/tp.2012.47; published online 29 May 2012

\section{Introduction}

Over the last decade, there has been increasing interest in the relationship between the circadian system and affective disorders. Various disruptions of circadian rhythms have been described in depressive disorders, including shifts in the onset and offset of the sleep phase relative to environmental time (phase-advance or delay), as well as disruption to the endogenous release of key hormones such as melatonin and cortisol and changes in core body temperature rhythm. ${ }^{1}$ Disruption to the rhythmicity of the systems under the control of the circadian system is likely to represent a fundamental disturbance of centrally regulated neurohormonal function, and may well underpin many of the somatic symptoms so often reported by patients with common mental disorders. ${ }^{2}$

There is evidence to suggest that sleep and/or circadian disturbance may be causally linked to both the emergence and persistence of affective disorders (see review by Harvey et al. ${ }^{3}$ and Wulff et al. ${ }^{4}$ ). Indeed, this notion is well supported by studies conducted in patients with seasonal affective disorder, where circadian misalignment has been linked with the onset, extent and resolution of depressive symptoms. ${ }^{5}$ Additionally, sleep-wake disturbance has been noted to be a prodromal, inter-episodic and prognostic feature of bipolar disorder (see review by Harvey et al. ${ }^{3}$ ). Misalignment of the circadian system relative to environmental time cues has profound affects not only on mood, but also on cognition, and a range of other physiological systems under circadian control, including thermoregulatory, endocrinological, immunological, cardiovascular and metabolic systems. ${ }^{4,6,7}$ Together, these data suggest that changes in the circadian system may not only represent a potential biomarker for illness onset and progression but may also be associated with adverse health and psychosocial outcomes.

The pattern of somatic features, sleep disturbance, daytime fatigue and related anxiety and depressive symptoms that often emerges throughout the adolescent period indicates the need to focus more closely on the underlying physiology of the developing circadian system. ${ }^{8}$ Developmental changes in the sleep-wake and circadian systems are common in adolescents and young adults, with delayed sleep phase syndrome a common feature of adolescence (see review by Crowley et al. ${ }^{9}$ ). It has been postulated that changes to both circadian timing and period are explained largely by intrinsic biological drives, rather than extrinsic environmental or psychosocial factors. ${ }^{9}$

As altered sleep patterns may precede the onset and persistence of psychological distress in young people, ${ }^{10}$ better characterization of these features may lead to identification of vulnerability markers that can then underpin better targeting of early interventions. ${ }^{4,11}$ To date, there has been little attempt to characterize these features in those at high risk or during the onset phases of affective disorders. ${ }^{4}$ Such objectives can, however, be accomplished by utilizing novel clinical staging

\footnotetext{
${ }^{1}$ Clinical Research Unit, Brain \& Mind Research Institute, University of Sydney, Camperdown, NSW, Australia and ${ }^{2}$ Chronobiology \& Sleep, Faculty of Arts, Business, Informatics \& Education, School of Management \& Marketing, Institute for Health and Social Science Research, Central Queensland University, Mackay, QLD, Australia Correspondence: Associate Professor SL Naismith, Clinical Research Unit, Brain \& Mind Research Institute, University of Sydney, Level 4, 94 Mallett Street, Camperdown, NSW 2050, Australia.

E-mail: sharon.naismith@sydney.edu.au

${ }^{3}$ Joint last author.

Keywords: affective; circadian; depression; melatonin; sleep; youth

Received 6 December 2011; revised 5 April 2012; accepted 10 April 2012
} 
paradigms seeking to identify young people in early stages of major mental disorders. ${ }^{11,12}$ Within this framework, young people presenting for care with admixtures of anxiety and depressive symptoms are typically categorized as being within early or 'attenuated syndrome' phases as compared with those with 'established disorders' (that is, first episode of major illness or later relapsing or persisting phases). ${ }^{12,13}$ Concurrently with the longitudinal evaluation of this model, we are testing whether there are distinct biomarkers evident at the different phases of affective illness.

The aim of the present study was to examine circadian parameters, notably the timing, secretion and synchrony of melatonin, according to the clinical stage of affective illness. ${ }^{12}$ Specifically, we aimed to examine melatonin onset and secretion patterns in those with early 'attenuated syndromes' as compared with those with 'established disorders'. It was hypothesized that those in later stages of illness would exhibit evidence of altered circadian functioning, in comparison with those in early illness phases.

\section{Subjects and methods}

Participants. A total of 44 young individuals were recruited from services that offer specialized assessment and early intervention of mental health problems in young people (Youth Mental Health Clinic (YMHC) at the Brain \& Mind Research Institute (BMRI); and headspace, Campbelltown, Sydney, Australia ${ }^{13,14}$ ). Inclusion criteria for this substudy were: (1) individuals aged 12-30 years seeking professional help primarily for significant anxiety or depressive symptoms; and (2) willingness to participate in overnight assessments of circadian rhythms (salivary melatonin) and sleep. Participants were excluded if they did not have sufficient English-language skills. The assessment protocol was approved by the University of Sydney Human Research Ethics Committee. Participants gave written informed consent before participation in the study.

Clinical assessments. As described elsewhere, ${ }^{12,13}$ patients entering the mental health services were assessed and managed by medically and/or psychologically trained health professionals. In this study, an independent psychiatrist or trained research psychologist conducted a standardized clinical interview, focussing on ratings of depressive symptom severity (Hamilton Depression Rating Scale (HDRS)) ${ }^{15}$ as well as assessment of the detailed criteria developed for formal application of our clinical staging framework. ${ }^{12}$ The clinical stage of affective disorder was rated by two psychiatrists with extensive clinical and research expertise in affective disorders and staging paradigms (ES and $\mathrm{IH}$ ). Patients were rated as having either an 'attenuated syndrome' (stage 1b) or an 'established disorder' (stage 2 and above). Within this staging system, patients may also be classified as being in 'stage 1a'. However, the stage 1a group is much more heterogeneous; although they are help seeking, with mild symptoms, there is subsequently less confidence that such subjects would transition to full-threshold disorders (see Hickie et al. ${ }^{12}$ ). Classification at stage 2 or above depends on the recognition of depressive syndromes with more severe features (for example, agitation, psychomotor retardation, psychotic features and additional intermittent hypomanic features). Such disorders may traditionally be classified as meeting full-threshold criteria for major depression according to a DSM-IV (Diagnostic and Statistical Manual of Mental Disorders, Fourth Edition) or ICD-10 (International Statistical Classification of Diseases and Related Health Problems, 10th Revision) diagnosis, and later stages are characterized predominantly by illness persistence or recurrence. Consequently, 52 and $56 \%$ of participants in our study who were classified as being in stage $1 \mathrm{~b}$ and stage $2+$, respectively, were taking antidepressant medications $\left(\chi^{2}=0.08\right.$, not significant (NS)). In comparison, $15 \%$ of those in stage $1 \mathrm{~b}$ were taking atypical antipsychotic medications as compared with $63 \%$ of those in stage $2+\left(\chi^{2}=10.4, P<0.01\right)$.

Sleep and circadian assessment. As described previously, ${ }^{16}$ participants completed diaries and/or actigraphy for 7 continuous days and nights before circadian assessment. Habitual sleep onset (HSO) was derived on a daily basis from a combination of light and activity data, which was supplemented by diary information, and averaged across the recording period. Participants then attended the Chronobiology and Sleep Laboratory at the BMRI for overnight circadian assessment. Participants were asked to arrive $7 \mathrm{~h}$ before their $\mathrm{HSO}$, to familiarize with the laboratory settings, and to ensure they were in a maintained posture for at least $30 \mathrm{~min}$ before the first sample being collected. As per standard dim light melatonin onset (DLMO) protocols, saliva samples were collected at 30-min intervals using Salivettes (Sarstedt, Germany) from $6 \mathrm{~h}$ before HSO until $2 \mathrm{~h}$ after HSO. That is, participants were kept awake $2 \mathrm{~h}$ past their HSO. At all times, while in the laboratory, participants were physiologically and behaviourally monitored under controlled conditions, with fixed light levels $(<50 \mathrm{Ix})$ and ambient temperature $\left(24 \pm 1^{\circ} \mathrm{C}\right)$. Participants maintained a seated posture for at least $20 \mathrm{~min}$ before each sample collection.

Melatonin was assayed in $200 \mu \mathrm{l}$ saliva by double antibody RIA (Cat no. RK-DSM2; Buhlmann Laboratories AG, Schönenbuch, Switzerland) according to the manufacturer's instructions. The lowest detectable level of melatonin was $4.3 \mathrm{pm}$. The intra-assay coefficient of variation was $<10 \%$ across the range of the standard curve. The inter-assay coefficient of variation was $15 \%$ at $19.5 \mathrm{pm}$ and $12.3 \%$ at $177 \mathrm{pM}$. The total area under the curve (AUC) was calculated using the trapezoid method, for each participant over the entire 8-h sampling period. To determine the DLMO, the average melatonin levels of the first three sampling times was calculated and a threshold of two standard deviations greater than this value was established for each subject. The DLMO was defined as the time when the saliva melatonin level first exceeded this threshold and remained elevated for at least the next sampling time. The phase angle of entrainment was calculated by subtracting the time of DLMO from HSO (measured in $\mathrm{min}$ ).

Self-report data. Patients were asked to complete the Beck Depression Inventory-II for depressive symptom severity. ${ }^{17}$ In order to assess daytime sleepiness in everyday or social 
situations, patients completed the Epworth Sleepiness Scale. ${ }^{18}$ Sleepiness during the evening of DLMO assessment was rated using the Karolinska Sleepiness Scale $^{19}$ (range $=1$ 'very alert' to 9 'extremely sleepyfighting sleep'). Ratings were obtained $3 \mathrm{~h}$ before HSO (KSS-3), and $1.5 \mathrm{~h}$ after HSO (KSS + 1.5).

Neuropsychological assessment. Within 2 weeks of laboratory assessment, a neuropsychologist administered the Trailmaking Test Part $A$ \& Part $B^{20}$ to assess psychomotor speed and set-shifting, respectively. Performance on this test was converted to a $z$-score according to age matched normative data. ${ }^{21}$ The Rey Auditory Verbal Learning Test $(\mathrm{RAVLT})^{22}$ was administered to measure unstructured verbal learning. Total learning over the five trials was used (RAVLTencoding, maximum $=75$ ) and scores were converted to $z$ scores according to age- and education-adjusted normative data. ${ }^{23}$ We specifically chose to examine these two measures as our prior work ${ }^{24,25}$ has shown them to be sensitive to underlying neurobiological changes in depressive disorders, even in young people at early stages of illness. ${ }^{26}$

Statistical analysis. Data were analysed using the Statistical Package for Social Sciences (SPSS version 19, IBM, Chicago, IL, USA). For continuous data, analyses employed Pearson's or Spearman's correlations where appropriate. For analyses between stage $1 \mathrm{~b}$ and stage $2+$, all categorical data were checked for distribution and normality. Student's $t$-tests were employed to analyse these data utilizing assumptions of equal or unequal variance where appropriate. All analyses were twotailed and used an $\alpha$ level of 0.05 .

\section{Results}

For three patients $(n=2$, stage $1 \mathrm{~b}$ and $n=1$, stage $2+)$, melatonin data were not observed (within the sensitivity of the assay) over the $8 \mathrm{~h}$ sampling period. For another three patients $(n=1$, stage $1 \mathrm{~b}$ and $n=2$, stage $2+)$, melatonin was detected but did not reach the threshold as required by the algorithm. Thus, DLMO and phase angle data were not available on a total of six patients. Demographic, clinical and DLMO data for the sample are presented in Table 1. There was no difference in the time of DLMO between the clinical stage groups. The stage $1 \mathrm{~b}$ and stage $2+$ groups did not differ statistically in terms of their level of subjective sleepiness $3 \mathrm{~h}$ before HSO and $1.5 \mathrm{~h}$ after HSO (KSS-3 and $\mathrm{KSS}+1.5)$, nor in their levels of daytime sleepiness, as assessed by the Epworth Sleepiness Scale. As expected, patients in earlier clinical stages were significantly younger; however, there was no difference in gender distribution. There was no significant difference in $\mathrm{HSO}$ in the week before assessment. However, stage $1 \mathrm{~b}$ patients had significantly greater phase angles than stage $2+$ patients when their DLMO was compared with their HSO times.

Analysis of salivary melatonin AUC data for patients in stage $1 \mathrm{~b}$ vs stage $2+$ showed that those in earlier stages had almost double the melatonin concentration of those in later stages (Table 1 and Figure 1). This was apparent for the entire sample as well as for melatonin concentration $2 \mathrm{~h}$ before and $2 \mathrm{~h}$ after HSO. Additionally, after controlling for age and depression
Table 1 Demographic, psychiatric and circadian data for patients with stage $1 \mathrm{~b}$ $(n=28)$ and $2+(n=16)$ affective disorders

\begin{tabular}{|c|c|c|c|}
\hline & $\begin{array}{l}\text { Stage 1b, } \\
\text { mean (s.d.) }\end{array}$ & $\begin{array}{l}\text { Stage 2+, } \\
\text { mean (s.d.) }\end{array}$ & t-value \\
\hline Age, years & $20.5(4.3)$ & $23.2(4.7)$ & $-2.2^{*}$ \\
\hline Sex, male/female ${ }^{a}$ & $15 / 13$ & $8 / 8$ & 0.2 \\
\hline Hamilton Depression Rating scale & $12.9(6.5)$ & $14.1(8.3)$ & -0.5 \\
\hline Education, years & $12.6(2.7)$ & $12.6(2.8)$ & -0.4 \\
\hline Beck Depression Inventory ${ }^{\mathrm{b}}$ & $18.0(8.6)$ & $24.8(11.1)$ & $-2.1^{*}$ \\
\hline Trailmaking Part $\mathrm{A}, \mathrm{z}$-score ${ }^{\mathrm{b}}$ & $-0.0(1.2)$ & $0.2(0.7)$ & -0.7 \\
\hline Trailmaking Part $\mathrm{B}, z$-score ${ }^{\mathrm{b}}$ & $-0.3(1.9)$ & $-0.4(1.2)$ & 0.3 \\
\hline RAVLT-encoding & $0.27(1.3)$ & $-0.48(1.4)$ & 1.7 \\
\hline Epworth Sleepiness scale & $6.4(3.7)$ & $7.1(4.1)$ & -0.6 \\
\hline Habitual sleep onset, time & $00: 34(01: 27)$ & $23: 56(01: 12)$ & 1.4 \\
\hline AUC, total sampled ${ }^{\mathrm{b}}$ & $142.3(85.7)$ & $69.3(60.4)$ & $2.7^{\star *}$ \\
\hline AUC, $2 \mathrm{~h}$ before $\mathrm{HSO}^{\mathrm{b}}$ & $51.5(38.8)$ & $20.3(22.0)$ & $3.3^{\star \star}$ \\
\hline $\mathrm{AUC}, 2 \mathrm{~h}$ after $\mathrm{HSO}^{\mathrm{b}}$ & $85.2(62.4)$ & $42.1(36.5)$ & $2.8^{*}$ \\
\hline DLMO, time & $21: 43(01: 41)$ & $22: 23(01: 37)$ & -1.2 \\
\hline Phase angle, min & $169.08(98.0)$ & $90.0(94.2)$ & $2.4^{*}$ \\
\hline KSS-3 & $5.6(2.1)$ & $6.8(1.6)$ & -1.9 \\
\hline $\mathrm{KSS}+1.5$ & $7.8(1.9)$ & $8.0(1.4)$ & -0.4 \\
\hline
\end{tabular}

Abbreviations: AUC, area under the curve; DLMO, dim light melatonin onset; KSS, Karolinska Sleepiness Scale; RAVLT, Rey Auditory Verbal Learning Test. ${ }^{\star} P<0.05,{ }^{* \star} P<0.01$. All test statistics are Student's $t$-test unless otherwise

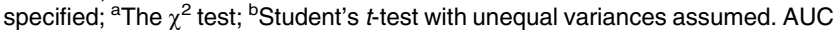
for melatonin over the sampling period (total sample), and for $2 \mathrm{~h}$ before and after habitual sleep onset; KSS $3 \mathrm{~h}$ before habitual sleep onset (KSS-3), and $1.5 \mathrm{~h}$ after habitual sleep onset (KSS+1.5). Note that DLMO data are missing for three patients in stage $1 \mathrm{~b}$ and three patients in stage $2+$ because of inability to detect melatonin within the sensitivity of the assay or not reaching the threshold as required by the algorithm.

severity, these analyses remained significant (total DLMO: $\mathrm{F}_{3,36}=4.9, \quad P<0.05$; DLMO $2 \mathrm{~h}$ before HSO: $\mathrm{F}_{3,36}=5.6$, $P<0.05$; and DLMO $2 \mathrm{~h}$ after $\left.\mathrm{HBO}: \mathrm{F}_{3,36}=4.6, P<0.05\right)$.

Correlations with depressive symptoms. There was no significant association between salivary melatonin AUC and either self-reported or clinician-rated depressive symptoms for patients with stage $1 \mathrm{~b}$ (Beck Depression Inventory (BDI), $r=-0.15$, NS; HDRS, $r=-0.15$, NS) or stage $2+(\mathrm{BDI}$, $r=0.12$, NS; HDRS, $r=0.37$, NS) affective disorders.

Correlation with subjective sleepiness. For both staging groups, there was no association between subjective sleepiness $3 \mathrm{~h}$ before $\mathrm{HSO}$, and total salivary melatonin AUC in the $3 \mathrm{~h}$ before HSO (KSS-3: $r=-0.17$ and $r=0.28$ for stage $1 \mathrm{~b}$ and $2+$, respectively). However, lower levels of salivary melatonin AUC $2 \mathrm{~h}$ after $\mathrm{HSO}$ were related to decreased levels of subjective sleepiness for those in stage $2+$ only (KSS + 1.5: $r=0.5, P<0.05$ and $r=0.29$, NS for stage $2+$ and $1 \mathrm{~b}$, respectively).

Correlation with cognition. For those in stage $1 \mathrm{~b}$, there were no significant relationships between total salivary melatonin AUC and performance on tasks of psychomotor speed (Trailmaking Part $A, r=0.12, N S$ ), new learning (RAVLT-encoding, $r=-0.04$, NS) or set-shifting (Trailmaking Part $B, r=-0.06, \mathrm{NS}$ ). For those in stage $2+$, lower levels of salivary melatonin AUC were not associated with processing speed or set-shifting (Trailmaking Part $A, r=0.46$, NS; Trailmaking Part $B, r=0.32, N S)$. However, as shown in Figure 2, lower melatonin levels were associated with poorer performance on a task of verbal memory encoding (RAVLTencoding, $r=0.58, P<0.05$ ). 


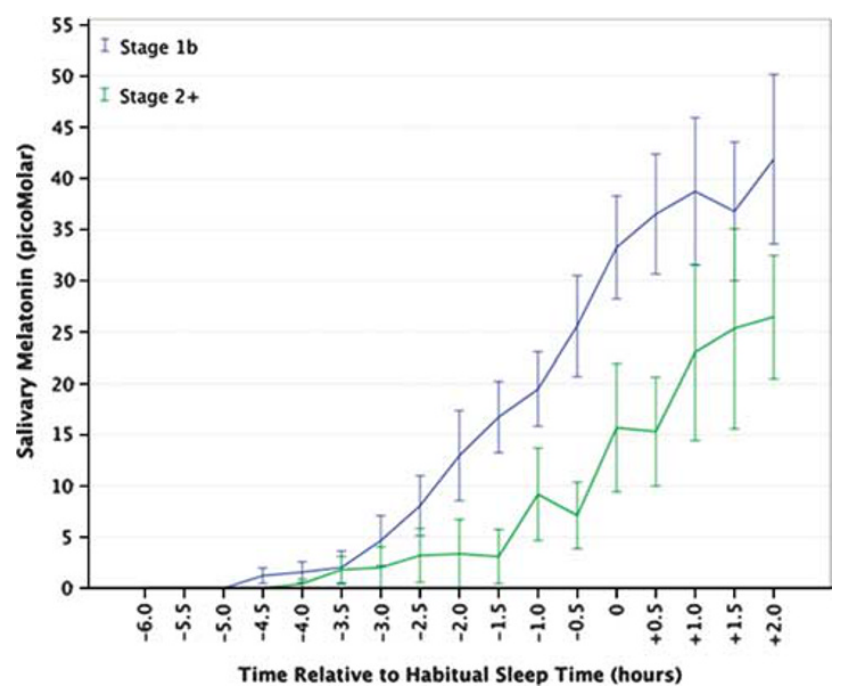

Figure 1 Graph demonstrating reduced salivary melatonin data (mean \pm s.e.m.) for patients with stage $2+$ affective disorders, relative to stage $1 \mathrm{~b}$. According to prior actigraphy monitoring, habitual sleep onset would normally occur at sample 0 .

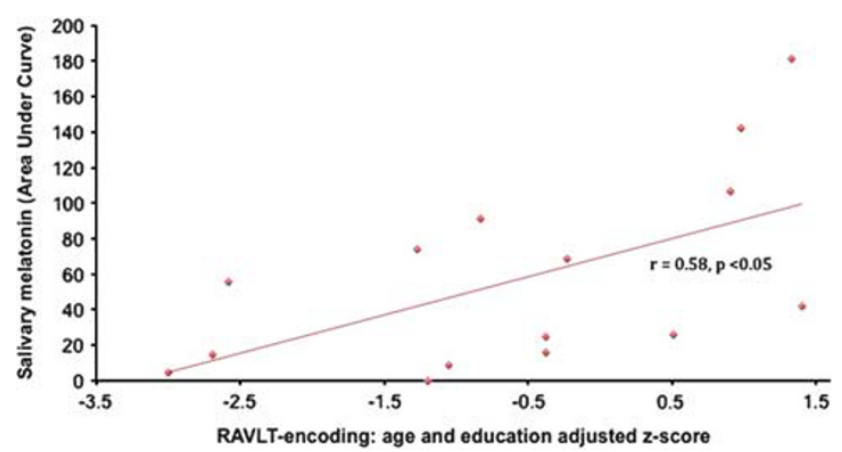

Figure 2 Scatterplot demonstrating the relationship between decreased salivary melatonin (area under curve) and memory performance (Rey Auditory Verbal Learning Test (RAVLT)-encoding trials) for patients with stage $2+(n=15)$ affective disorders. Note that neuropsychological assessment occurred within a 2week timeframe and did not occur in the evening of dim light melatonin onset (DLMO) assessment.

\section{Discussion}

The present study has demonstrated that young people in later stages (that is, 'established disorders') of major affective disorders have significantly lower salivary melatonin levels assessed during the first part of the melatonin secretory period than those who are still at earlier stages of illness (that is, 'attenuated syndromes'). In turn, lower salivary melatonin levels appeared to have functional correlates as they were associated with decreased ratings of sleepiness. This finding is consistent with previous studies describing the relationship between melatonin and sleepiness levels. ${ }^{27,28}$ The fact that this was more apparent in the time after HSO is expected, as this is when melatonin secretion would be elevated, although it is important to note that there may be more variability in melatonin levels during this time. In addition, the present study has shown that differences in melatonin secretion profiles are related to other markers of underlying neurobiology, namely neuropsychological functioning. Specifically, lower melatonin levels during the first part of the night were related to poorer performance on the encoding component of the RAVLT. Performance on this task of unstructured verbal learning (that is, word-list learning) is commonly compromised in patients with affective disorders, ${ }^{25,29}$ and in this sample, over twothirds of participants performed below average. As prior work has demonstrated that poor performance may reflect dysfunction in frontotemporal, including hippocampal circuitry, these preliminary data may suggest that disturbances of the circadian system and neuropsychological dysfunction may reflect abnormalities in common underlying neural circuitry. From these data, we cannot ascertain the temporal relationship between dysfunction in the sleep and cognitive systems, as neuropsychological testing did not occur at the same time. Future studies may focus on delineating these relationships further.

Regarding circadian timing, differences were not apparent between the clinical staging groups, with DLMO occurring on average at 2130 and $2200 \mathrm{~h}$ for those with 'attenuated syndromes' and 'established disorders', respectively. Interestingly, for those in later clinical stages, the timing of melatonin onset and habitual sleep onset occurred within the expected 120-min period, whereas for those with 'attenuated syndromes', the difference in the timing of these two systems was $\sim 3 \mathrm{~h}$. Although it is difficult to interpret these findings in the absence of a control group and full melatonin profile, these data may suggest that the circadian pacemaker is phase-shifted (advanced) relative to timing of the sleepwake cycle. Alternatively, the sleep-wake timing may be delayed, although it is noted that sleep onset is only weakly influenced by the circadian system, and delays in sleep may be due to a number of confounders. As we did not measure peak melatonin amplitude in our current protocol, we cannot ascertain whether there was attenuation in circadian amplitude in this group. However, this seems unlikely as those with 'attenuated syndromes' did not have reduced melatonin secretion (that is, the absolute amount of melatonin secretion was higher) relative to those with 'established disorders'. Conversely, another possible explanation for these findings could be that patients with 'established disorders' have a shortened phase angle or even a phase delay, relative to those at early stages of illness. As stated above, in the absence of a control group and full melatonin profile, further studies are now required to delineate these possibilities. It is also worth noting that a greater proportion of those with 'established disorders' were taking antipsychotic medications. Although little is known about the effect of atypical antipsychotics on circadian parameters, a preliminary study has suggested that the older 'typical' antipsychotics have disruptive effects on circadian rhythms. ${ }^{30}$ Thus, we cannot rule out the possible contribution of antipsychotic medication to the disturbances in circadian parameters observed within this study.

In terms of underlying circadian regulation of the sleepwake cycle, it is clear that the circadian clock in the anterior hypothalamus is critical for establishing the circadian rhythm of sleep-wake behaviour. ${ }^{31}$ However, the suprachiasmatic nucleus itself has only minimal monosynaptic outputs to sleep-regulation centres such as the ventrolateral preoptic nucleus and the lateral hypothalamus and has no outputs to 
brainstem arousal sites. ${ }^{31}$ Thus, the weakened relationship between the sleep and circadian systems in early-stage patients (that is, as suggested by increased phase angles) may reflect alterations in a multiple range of divergent pathways. Indeed, recent models regarding synchrony of the sleep and circadian systems highlight the critical role of the dorsomedial hypothalamic nucleus. The dorsomedial hypothalamic nucleus sends a glutamatergic projection to the lateral hypothalamus (overlapping with the field of orexincontaining neurons) as well as a GABAergic projection to the ventrolateral preoptic nucleus. ${ }^{31}$ Thus, desynchronization between the sleep and circadian system may be because of increased wake promotion via orexin neurons (resulting in a delayed sleep phase despite homeostatic pressure) or a reduction in amplitude of the circadian signal reaching the ventrolateral preoptic nucleus. ${ }^{32}$ As the dorsomedial hypothalamic nucleus is very sensitive to self-imposed schedules, and integrates circadian signals with environmental and social factors, behavioural feedback is recognized to be a critical influence in sleep-wake patterns particularly to light exposure and meal times. In this study, we cannot attribute the findings regarding melatonin secretion and sleep timing in patients with 'attenuated syndromes' to any particular process; therefore, further empirical studies specifically examining the synchrony between these systems are now warranted.

Overall, these data suggest that with the emergence of major mood disorders in young people, fundamental changes in the sleep and circadian systems are occurring that are not merely because of pubertal development, as commonly seen in 'healthy' adolescents. Importantly, they highlight that interventions targeting the circadian system are warranted even in early phases of illness where there appears to be some degree of misalignment of the sleep and circadian systems. As more marked dysfunction in the circadian system was observed in those with 'established disorders', these data suggest that persistence of depressive symptoms may perpetuate disruptions within the circadian system. Conversely, persistent sleep-wake disturbance in young people may contribute to ongoing psychological distress, an observation that has been reported recently from epidemiological data. ${ }^{10}$ In either case, these data indicate that differential interventions may be required for those presenting at different clinical stages. Specifically, behavioural interventions targeting depressive symptoms and sleep-wake functioning concurrently appear warranted in those with 'attenuated syndromes' and sleep-wake disturbance. In 'established disorders', clinical assessment should incorporate circadian markers (for example, melatonin, core body temperature) where possible and interventions may be much more targeted in order to address the lowered levels of melatonin likely to be observed in this group. Such agents may incorporate the use of pharmacological compounds such as melatonin, melatonin analogues or the newer antidepressants targeting both mood and sleep symptoms (see Hickie and Rogers ${ }^{1}$ for a review). Although at this stage it is unclear whether circadian misalignment is causally linked to cognitive dysfunction, or merely co-occurs with neurobiological changes observed with the disease, it is possible that improvements in both the mood and sleep-wake systems will have broader benefits for both cognition and functional outcomes. In this regard, it is worth noting that the decreased melatonin observed in this group was only weakly associated with depressive symptom severity, suggesting that interventions aiming to improve functioning should target more than depressive symptoms alone.

Although the current study represents the first to examine circadian rhythms in young people with emerging affective disorders, some limitations exist. First, as expected, those in later stages had more severe disorders and, consequently, greater numbers of patients had psychotic or hypomanic features, and psychotropic use was more common in this group. Second, although in the present study we measured melatonin secretion under appropriate dim light conditions, studies have shown that the timing of melatonin onset can be influenced by light exposure the prior day. ${ }^{33}$ Thus, some variability between groups in terms of light exposure may have influenced these findings. Third, as this was not a longitudinal study, we are uncertain whether patients move from a weakened sleep-wake and circadian relationship early in the course of disorder to a more compensated shift in sleepwake cycle (that is, with later sleep-onset (phase-delay) and resynchronizing with the circadian rhythm).

In conclusion, this study presents the first preliminary findings suggesting that melatonin may be a viable marker of affective disease progression and may assist with personalized treatment planning. Future research may extend these findings by examining the predictive utility of melatonin as a biomarker for disease progression. It may also examine whether individually tailored chronobiological interventions provide more optimal treatment outcomes for young people with affective disorders than conventional treatment approaches.

\section{Conflict of interest}

$\mathrm{IBH}$ has led projects for health professionals and the community supported by governmental, community agency, and drug industry partners (Wyeth, Eli Lily, Servier, Pfizer, Astra Zeneca) for the identification and management of depression and anxiety. He has served on advisory boards convened by the drug industry in relation to specific antidepressants, including nefazodone, duloxetine, and desvenlafaxine, and has participated in a multicenter clinical trial of agomelatine effects on sleep architecture in depression. He has participated in Servler-sponsored educational programs related to circadian-based therapies.

Acknowledgements. This study was funded from an NHMRC Australia Fellowship awarded to Professor lan Hickie. Associate Professor Sharon Naismith is funded by an NHMRC Career Development Award. We acknowledge Associate Professor David Kennaway for expertise with melatonin analysis.

1. Hickie IB, Rogers NL. Novel melatonin-based therapies: potential advances in the treatment of major depression. Lancet 2011; 378: 621-631.

2. Hickie IB, Davenport TA, Scott EM, Hadzi-Pavlovic D, Naismith SL, Koschera A. Unmet need for recognition of common mental disorders in Australian general practice. Med J Aust 2001; 175(Suppl): S18-S24.

3. Harvey AG, Mullin BC, Hinshaw SP. Sleep and circadian rhythms in children and adolescents with bipolar disorder. Dev Psychopathol 2006; 18: 1147-1168.

4. Wulff K, Gatti S, Wettstein JG, Foster RG. Sleep and circadian rhythm disruption in psychiatric and neurodegenerative disease. Nat Rev Neurosci 2010; 11: 589-599. 
5. Lewy AJ. Circadian misalignment in mood disturbances. Curr Psychiatry Rep 2009; 11: 459-465.

6. Czeisler $\mathrm{C}$, Buxton $\mathrm{O}$, Khalsa $\mathrm{S}$. The human circadian timing system and sleep-wake regulation. In: Kryger MH, Roth T, Dement WC (eds). Principles and Practice of Sleep Medicine 4th edn. W. B. Saunders Company: Philadelphia, 2005, pp. 375-394.

7. Dijk DJ, Lockley SW. Functional genomics of sleep and circadian rhythm. Invited review: integration of human sleep-wake regulation and circadian rhythmicity. J Appl Physiol 2002; 92: 852-862.

8. Hansell NK, Wright MJ, Medland SE, Davenport TA, Wray NR, Martin NG et al. Genetic comorbidity between neuroticism, anxiety/depression and somatic distress in a population sample of adolescent and young adult twins. Psychol Med 2011; 42: 1-12.

9. Crowley SJ, Acebo C, Carskadon MA. Sleep, circadian rhythms, and delayed phase in adolescence. Sleep Med 2006; 8: 602-612.

10. Glozier N, Martiniuk A, Patton G, Ivers R, Li Q, Hickie I et al. Short sleep duration in prevalent and persistent psychological distress in young adults: the drive study. Sleep 2010; 33: 1139-1145

11. Mcgorry PD, Hickie IB, Yung AR, Pantelis C, Jackson HJ. Clinical staging of psychiatric disorders: a heuristic framework for choosing earlier, safer and more effective interventions. Aust N Z J Psychiatry 2006; 40: 616-622.

12. Hickie IB, Scott EM, Hermens DF, Naismith SL, Guastella AJ, Kaur M et al. Applying a clinical staging framework in young people who present with admixtures of anxious, depressive or psychotic symptoms. Early Interv Psychiatry (in press).

13. Scott EM, Hermens DF, Glozier N, Naismith SL, Guastella AJ, Hickie IB. Targeted primary care-based mental health services for young Australians. Med J Aust 2012; 196: 136-140.

14. Scott E, Naismith S, Whitwell B, Hamilton B, Chudleigh C, Hickie I. Delivering youthspecific mental health services: the advantages of a collaborative, multi-disciplinary system. Australas Psychiatry 2009; 17: 189-194.

15. Hamilton M. A rating scale for depression. J Neurol Neurosurg Psychiatry 1960; 23: 56-62.

16. Naismith SL, Rogers NL, Lewis SJ, Terpening Z, Ip T, Diamond K et al. Sleep disturbance relates to neuropsychological functioning in late-life depression. J Affect Disord 2011; 132 139-145.

17. Beck AT, Steer RA, Ranieri WF. Manual for Beck Depression Inventory-II. Psychological Corporation: San Antonio, TX, 1996.

18. Johns MW. A new method for measuring daytime sleepiness: the Epworth sleepiness scale. Sleep 1991; 14: 540-545.

19. Akerstedt T, Gillberg M. Subjective and objective sleepiness in the active individual. Int $J$ Neurosci 1990; 52: 29-37.

20. Reitan RM. Manual for Administration for Neuropsychological Test Batteries for Adults and Children. Reitan Neuropsychological Laboratory: Tucson, AZ, 1979.
21. Tombaugh TN. Trail Making Test A and B: normative data stratified by age and education. Arch Clin Neuropsychol 2004; 19: 203-214.

22. Spreen O, Strauss E. A Compendium of Neuropsychological Tests: Administration, Norms, and Commentary. 2nd edn. Oxford University Press: New York, 1998.

23. Senior G. Analysing RAVLT Learning and Serial Position Curves Using Mahalanobis Distance. University of Southern Queensland: Toowoomba, QLD, Australia, 1999.

24. Naismith S, Hickie I, Ward PB, Turner K, Scott E, Little $\mathrm{C}$ et al. Caudate nucleus volumes and genetic determinants of homocysteine metabolism in the prediction of psychomotor speed in older persons with depression. Am J Psychiatry 2002; 159: 2096-2098.

25. Hickie IB, Naismith S, Ward PB, Turner K, Scott E, Mitchell P et al. Reduced hippocampa volumes and memory loss in patients with early- and late-onset depression. Br J Psychiatry 2005; 186: 197-202.

26. Hermens DF, Naismith SL, Redoblado Hodge MA, Scott EM, Hickie IB. Impaired verba memory in young adults with unipolar and bipolar depression. Early Interv Psychiatry 2010; 4: 227-233.

27. Cajochen C, Krauchi K, Von Arx M-A, Mori D, Graw P, Wirz-Justice A. Daytime melatonin administration enhances sleepiness and theta/alpha activity in the waking EEG. Neurosci Lett 1996; 207: 209-213.

28. Krauchi K, Cajochen C, Pache M, Flammer J, Wirz-Justice A. Thermoregulatory effects of melatonin in relation to sleepiness. Chronobiol Int 2006; 23: 475-484.

29. Hermens DF, Redoblado Hodge MA, Naismith SL, Kaur M, Scott E, Hickie IB. Neuropsychological clustering highlights cognitive differences in young people presenting with depressive symptoms. J Int Neuropsychol Soc 2011; 17: 267-276.

30. Wirz-Justice A, Haug HJ, Cajochen C. Disturbed circadian rest-activity cycles in schizophrenia patients: an effect of drugs? Schizophr Bull 2001; 27: 497-502.

31. Fuller PM, Gooley JJ, Saper CB. Neurobiology of the sleep-wake cycle: sleep architecture, circadian regulation, and regulatory feedback. J Biol Rhythms 2006; 21: 482-493.

32. Phillips AJK, Czeisler CA, Klerman EB. Revisting spontaneous internal desynchrony using a quantitative model of sleep physiology. J Biol Rhythms 2011; 26: 441-453.

33. Chang AM, Scheer FA, Czeisler CA. The human circadian system adapts to prior photic history. J Physiol 2011; 589: 1095-1102.

Translational Psychiatry is an open-access journal published by Nature Publishing Group. This work is licensed under the Creative Commons Attribution-Noncommercial-No Derivative Works 3.0 Unported License. To view a copy of this license, visit http://creativecommons.org/licenses/by-nc-nd/3.0/ 